\title{
Genomics in analysis of Chlamydia psittaci host-adaptation
}

\author{
O. Voronina*, M. Kunda, E. Aksenova, N. Sharapova, N. Ryzhova, N. Bondareva, \\ N. Zigangirova \\ N.F. Gamaleya National Research Center for Epidemiology and Microbiology, Moscow, Russia \\ *e-mail:olv550@gmail.com
}

Key words: Chlamydia psittaci, polymorphic membrane proteins, plasticity zone, type III secretion system

Motivation and Aim: Chlamydia psittaci is an economically relevant pathogen in livestock and pet animals, where it causes psittacosis/ornithosis, and also a human pathogen causing atypical pneumonia after zoonotic transmission. Conjunctivitis, lung diseases, enteritis, animals aborted fetus are associated with this pathogenic bacteria. Earlier we proved that three Chlamydia strains, isolated from synovial fluid of patients with reactive arthritis were $C$. psittaci. Genome sequencing of these strains (Accession numbers CP024451, CP024453, CP024455, ST (sequence type) 24) revealed that they are closely related. The main zones of variability in very conservative Chlamydia genomes are plasticity zone (PZ) and zones of polymorphic membrane proteins (pmp). These zones of the synovial strains had the highest similarity with 01DC11 strain isolated from pig conjunctiva (ST24). Extending these strains investigation we analyzed type III secretion system (T3SS) as main pathogenicity factor participating in host-microbe interactions. Methods and Algorithms: RAST v.2.0 server was used for genome annotation; TMHMM Server v.2.0 - for prediction of protein localization; Virulence Factor DataBase and EffectiveDB (EffectiveT3) - for revealing the genes associated with T3SS and its effectors; COILS server - for prediction of helix coiled-coil domains.

Results: Analysis of the synovial strains genomes revealed 62 genes coding intact proteins and effectors of T3SS that is able to inject proteins directly into cytoplasm of the eukaryotic cells. Only few groups of these genes were organized in the operons, in contrast with Betaproteobacteria having one big T3SS gene cluster. Among ST24 strains of the animal origin we found only 3 genes which were differed from synovial T3SS genes by 1-2 mismatches. Human ST24 strain from bronchial lavage had 3-4 mismatches in the other 3 genes. In contrast, ST35 strain isolated from throat of humans with an influenzalike infection demonstrated differences in $79 \%$ of T3SS genes. The most number of changes, 94 mismatches and 2 gaps, were in the gene of putative cytotoxin. The genes coding proteins of the export apparatus were predominant among the genes with $100 \%$ similarity. There were only two genes of the effectors in this group. Moreover among 28 pmp genes of ST35 strain there was only one with $100 \%$ similarity with pmp genes of the synovial strains.

Conclusion: . psittaci ST24 strains are wide spread, polyhostal, adapted to the wide host range, including birds, livestock animals and human, and are able to infect the cell of the different tissue, causing the large list of the diseases. PZ, pmp, T3SS genes of ST24 strains are significantly differ from the genes of the strains with the rare ST. These data allow to elaborate common medicine for eradication of $C$. psittaci ST24 strains. Acknowledgements: Supported by the State Order to the N.F. Gamaleya National Research Center for Epidemiology and Microbiology. 